\title{
Performance Analysis of a Novel Dual-Frequency Multiple Access Relay Transmission Scheme
}

\author{
Javier DEL SER ${ }^{1 *}$, Babak H. KHALAJ ${ }^{2}$ \\ ${ }^{1}$ TECNALIA-TELECOM, 48170 Zamudio-Bizkaia, Spain. \\ ${ }^{2}$ Department of Electrical Engineering, Sharif University of Technology, Tehran, Iran. \\ Email:jdelser@robotiker.es,khalaj@sharif.ir \\ Received June 26, 2009; revised July 31, 2009; accepted August 27, 2009
}

\begin{abstract}
In this paper we present the performance analysis of a novel channel assignment scheme where two non-cooperative independent users simultaneously communicate with their destination through a single relay by using only two frequency channels. The analytic derivation of the probability of symbol error for two main relay techniques will be provided, namely Amplify-and-Forward (AF) and Decode-and-Forward (DF). As shown by the obtained results, our switched-frequency approach results in a model that can achieve fulldiversity by means of maximum-likelihood decoding at the receiver. Our results are especially important in the DF case, since in traditional techniques (such as half-duplex two-time slot approaches) two sources simultaneously transmit on the same channel through the first time slot, which necessitates some sort of superposition coding. However, since in our scheme both users transmit over orthogonal channels, such a coding scheme is not required. In addition, it is shown that the DF approach based on our novel channel assignment scheme outperforms the AF scheme, especially in scenarios where the relay is closer to the receiver.
\end{abstract}

Keywords: Multiple Access Relay Channel, Frequency Switching, Non-Cooperative Networks, Maximum Likelihood Decoding

\section{Introduction}

During the last years the use of relay nodes has attracted a lot of attention in practical areas such as cellular networks, especially in scenarios where multiple antennas cannot be installed in practice at any site. Deploying relay nodes between sender(s) and receiver(s) provides increased spatial diversity in the communication scenario under consideration. The research community has shown a great interest in this field: as to mention, for the single user scenario with relay channels, capacity bounds are computed for Detect-and-Forward (DF) based mechanism in a Rayleigh fading environment [1,2]. The outage capacity bounds for the case of a single user transmission with relay in low signal-to-noise ratio regime is considered in [3], in which frequency division model for the relay channel is assumed. The authors in [4] present the performance limits of Amplify-and-Forward (AF) relay channels for single user scenario, where a new transmission protocol is also proposed in order to achieve full diversity. Recently, the use of practical coding schemes at the relay has also been addressed in $[5,6]$.
In this context, this paper will focus on the performance analysis of a non-cooperative two-source multiple access relay channel (MARC) [7]. In the MARC channel, two independent information sources transmit their data to a common destination aided by a shared relay node, which processes and combines the data from both sources. The achievable rate region for the MARC channel has been studied in [8] by employing a partial detect-and-forward strategy at the relay. In multiuser scenarios, cooperative ideas have also been proposed, so that users could interact with each other in order to improve each other's performance in fading environments [9-13]. In [14], the authors present an upper bound on the diversity-multiplexing trade-off for the single user relay channel. However, their proposed scheme does not achieve full diversity for the whole block transmission period, since samples in the second transmission slot are not protected by relay re-transmissions. The authors also extend their scheme to cooperative multiple access scenarios for the two user case in the absence of any additional relays. However, since such cooperative schemes rely on an inter-user channel which consumes network 
resources and therefore limits their performance by the condition of such a link [15], in this paper we will only focus on non-cooperative multiple access channels.

Traditionally, the case of multiple users and a relay has been addressed in a number of different ways. The most straightforward approach is to extend single-relay ideas to multiple users, in which each user uses a separate frequency and diversity is achieved by utilizing two time-slots. Such an approach is basically an extension of the well-known delay diversity schemes [16,17] and [18], which will naturally lead to a total of four orthogonal time-frequency channels. Another approach is to consider independent receive channels for signals coming from the users and the relay, also resulting in a total of four independent channels either in time or frequency. It should be especially noted that, although it is possible to extend half-duplex two-time slot Amplify-and-Forward schemes to MARC scenarios [19], the extension of Detect-and-Forward schemes to MARC requires the use of complex superposition-type multiuser coding strategies, so that the relay is able to detect multiple sources over the same multiple access channel $[8,19,20,21]$.

The scheme proposed in this paper does not rely on any special coding scheme, since the users transmit to the relay over orthogonal channels. Our analysis will be focused on examining the error performance of the MARC scenario in the case of full multiplexing gain (two independent sources transmitting simultaneously in the same time slot), where diversity gain improvement can be verified through the slope of error probability curve as a function of received SNR in a log-log scale. As will be shown, by using the proposed frequencyswitching channel assignment scheme at the relay (Figure 1), the signal model will be transformed from two independent scalar channels into a $2 \times 2$ Multiple-Input Multiple-Output (MIMO) model [22]. Consequently, Maximum Likelihood (ML) detection performed over the received vector will provide an overall diversity order of two for each user.

The structure of the paper is organized as follows. In Section 2, the signal model and the channel assignment scheme will be presented. In addition to Amplify-andForward scheme, Detect-and-Forward structure and its corresponding ML detector will be proposed. In Section 3, analytic probability of error computations for both AF and DF schemes will be derived. In Section 4, simulation results for both AF and DF mechanisms for different relay locations will be presented and compared with the analysis. As shown in these results, the DF approach outperforms the AF scheme, especially as the relay gets closer to the destination. Finally, Section 5 concludes the paper.

\section{Signal Model}

In this paper, we will assume the case of two independent and identically distributed (i.i.d.) random sources $S_{I}$ and $S_{2}$ which generate binary symbols $b_{1}$ and $b_{2}\left(b_{1}, b_{2} \in\right.$ $\{-1,1\})$, respectively. Those symbols are transmitted in a wireless environment to the same destination. In between these sources and the destination a single dualfrequency relay is located. The distance between sources and destination is normalized to one, and the location of the relay is denoted as $d(0<d<1)$. The channel is assumed to be block Rayleigh fading, i.e. the channel is assumed to be fixed within a block and varies independently from block to block. In addition to this Rayleigh model, a propagation loss as a function of distance is considered. This loss is a basic exponential model with exponent $n=2$, hence the power attenuation is assumed to be equal to $K / d^{2}$ at a distance $d$, where $K$ is the propagation constant [23]. The channel state information (CSI) is only assumed to be known at the receiver locations (i.e. both relay and destination), whereas the transmitters are not assumed to have any knowledge of their forward transmission channels. Both users transmit their signals at two different frequency channels $f_{1}$ and $f_{2}$, respectively.

Instead of forwarding each received signal over the same incoming frequency channel, the relay of our proposed dual-frequency channel assignment switches the frequencies between the two transmitted signals. The proposed structure is shown in Figure 1, where the source $S_{1}$ transmits at frequency channel $f_{1}$, and the relay retransmits the same signal over frequency channel $f_{2}$. Similarly, the frequency channel of source $S_{2}$ is switched at the relay before retransmission to the destination. It can be easily verified that, without such frequency switching at the relay, no additional diversity can be achieved without resorting to delay diversity schemes, since the two signals coming from the source and the relay at each frequency are simultaneously combined at the receiver. As will be subsequently shown, the proposed channel assignment scheme will transform two independent scalar channels into a two dimensional vector channel which achieves a diversity of order two.

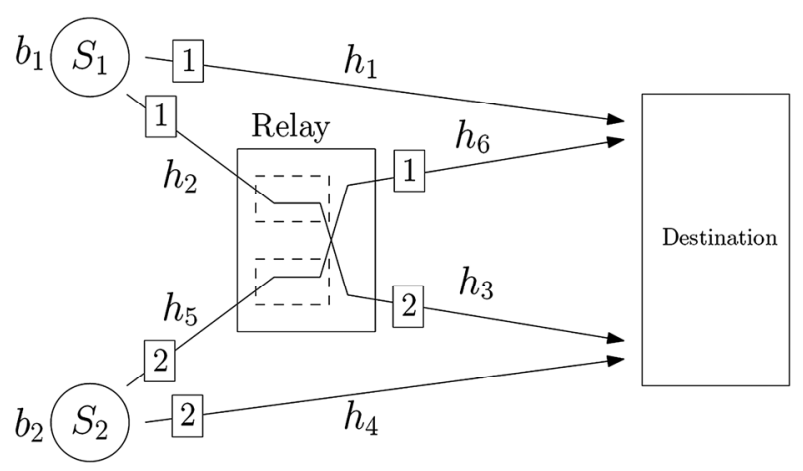

Figure 1. The proposed switched frequency assignment at the relay (the number over each link denotes the corresponding frequency channel used). 
It should be noted that in the proposed scheme, the relay should perform in a full-duplex mode for each transmission path. In other words, two transceivers should operate simultaneously in the same time slot at the relay. However, since these two transceivers can be stationed separately in hardware and the input carrier frequency of each board is different from its output carrier frequency, the hardware complexity will be significantly less than the traditional full-duplex transceivers that operate on the same carrier frequency for both their input and output signals. In addition, the aforementioned assumption would lead to echo-interference in case of highly asymmetric receive and transmit power. Such an issue could be overcome by applying preprocessing and postprocessing techniques as done, for instance, in [24]. Nevertheless, the echo interference will be assumed to be negligible at the relay, since its suppression falls out of the scope of our contribution.

In the next subsections we present the two previously mentioned relaying schemes, DF and AF, particularized for our proposed setup.

\subsection{Mplify-and-Forward ML Detector at the Relay}

Assuming that signals transmitted by each source are denoted by $b_{1}$ and $b_{2}$, and that all information symbols from source and relay stations reach the common destination in the same time slot (as done, for instance, in [25, $5]$ ), the received signal for each frequency will be given by

Frequency channel $f_{1}$ :

$$
y_{1}=b_{1} h_{1}+\left(b_{2} h_{5}+n_{2}\right) \gamma_{1} h_{6}+n_{3}
$$

Frequency channel $f_{2}$ :

$$
y_{2}=b_{2} h_{4}+\left(b_{1} h_{2}+n_{1}\right) \gamma_{2} h_{3}+n_{4}
$$

where $h_{1}$ and $h_{4}$ denote the channel coefficients between sources $S_{1}$ and $S_{2}$ and the destination, $h_{2}$ and $h_{5}$ denote channel taps between sources $S_{1}$ and $S_{2}$ and the relay, and $h_{3}$ and $h_{6}$ denote the channel weights between relay and destination at the two transmit frequencies $f_{1}$ and $f_{2}$, correspondingly. All channel coefficients $\left\{h_{i}\right\}_{i=1}^{6}$ are modeled as complex Gaussian random variables with zero mean and variance per dimension equal to $K / d^{2}$ (due to the propagation loss at the distance $d$ ). In addition, complex circularly symmetric zero mean additive white Gaussian noise $\left\{n_{i}\right\}_{i=1}^{4}$ are assumed for receive inputs at both relay and destination, with variance per dimension $\sigma^{2}=N_{0} / 2$. Also, the quantities $\gamma_{1}$ and $\gamma_{2}$ denote the gain of the relay for each frequency channel $f_{1}$ and $f_{2}$, whose values are chosen such that the relay transmits with unit average power.

A closer look at the above equations reveals that, by the proposed switching algorithm at the relay, the channel is transformed into a $2 \times 2$ MIMO channel model as given by

$$
\begin{aligned}
\left(\begin{array}{l}
y_{1} \\
y_{2}
\end{array}\right) & =\left(\begin{array}{cc}
h_{1} & \gamma_{1} h_{5} h_{6} \\
\gamma_{2} h_{2} h_{3} & h_{4}
\end{array}\right)\left(\begin{array}{l}
b_{1} \\
b_{2}
\end{array}\right) \\
& +\left(\begin{array}{l}
\gamma_{1} h_{6} n_{2}+n_{3} \\
\gamma_{2} h_{3} n_{1}+n_{4}
\end{array}\right)
\end{aligned}
$$

As is well-known in MIMO literature, the above model can be solved in the context of standard spatial multiplexing MIMO systems, where the detection based on the Maximum Likelihood (ML) criterion yields a diversity of order two [26]. Consequently, in our approach we use a ML detector at the destination that will jointly estimate $b_{1}$ and $b_{2}$ from the received signals $y_{1}$ and $y_{2}$. It should also be observed that in our model, some entries of the channel matrix shown in Equation (3) are multiplications of Rayleigh variables, and therefore the resulting model is not roughly in the conventional form spatial multiplexing models. Nevertheless, the obtained results show that an increased diversity gain of order close to two will still be achieved for both users in the DF approach.

\subsection{Detect-and-Forward ML Detector at the Relay}

Instead of just amplifying and forwarding each signal, in this case the relay detects the source symbol from the received signal before retransmitting it. Consequently, in the DF approach the received signal at destination will be given by

Frequency channel $f_{1}$ :

$$
y_{1}=b_{1} h_{1}+\hat{b}_{2} \gamma_{1} h_{6}+n_{3}
$$

Frequency channel $f_{2}$ :

$$
y_{2}=b_{2} h_{4}+\hat{b}_{1} \gamma_{2} h_{3}+n_{4}
$$

where $\hat{b}_{1}$ and $\hat{b}_{2}$ denote the output of the detector at the relay for sources $S_{1}$ and $S_{2}$, respectively.

It should be noted that in this case, the maximum likelihood detector at the destination should also consider the effect of detection errors at the output of the relay. Such errors are mainly due to fading events in the source-relay links: when one of these links is affected by a deep fade, the detection errors committed at the relay are propagated to the destination. In order to account for both source-relay and relay-destination links, an end-to-end ML detector should be utilized. The associated end-toend search criterion can be derived by first modeling the source-relay link as a binary symmetric channel (BSC) with probability of error equal to 


$$
\begin{aligned}
& P_{e}^{1}=Q\left(\frac{\left|h_{2}\right|}{\sigma}\right) \\
& P_{e}^{2}=Q\left(\frac{\left|h_{5}\right|}{\sigma}\right)
\end{aligned}
$$

where $Q(\bullet)$ denotes the standard $Q$-function. The estimated $b_{1}$ and $b_{2}$ values at the final destination, denoted by $\tilde{b}_{1}$ and $\tilde{b}_{2}$, are then computed by maximizing the likelihood function

$$
\begin{aligned}
& \left(\tilde{b}_{1}, \tilde{b}_{2}\right)=\underset{b_{1}, b_{2} \in\{-1,1\}}{\operatorname{argmax}} p\left(y_{1}, y_{2} \mid b_{1}, b_{2}\right) \\
& =\underset{b_{1}, b_{2} \in\{-1,1\}}{\operatorname{argmax}} \sum_{\hat{b}_{1}, \hat{b}_{2}} p\left(y_{1}, y_{2} \mid b_{1}, b_{2}, \hat{b}_{1}, \hat{b}_{2}\right) p\left(\hat{b}_{1}, \hat{b}_{2} \mid b_{1}, b_{2}\right.
\end{aligned}
$$

where

$$
\begin{aligned}
& p\left(\hat{b}_{1}, \hat{b}_{2} \mid b_{1}, b_{2}\right) \\
& = \begin{cases}\left(1-P_{e}^{1}\right)\left(1-P_{e}^{2}\right) & \text { if } \hat{b}_{1}=b_{1}, \hat{b}_{2}=b_{2} . \\
P_{e}^{1}\left(1-P_{e}^{2}\right) & \text { if } \hat{b}_{1} \neq b_{1}, \hat{b}_{2}=b_{2} . \\
\left(1-P_{e}^{1}\right) P_{e}^{2} & \text { if } \hat{b}_{1}=b_{1}, \hat{b}_{2} \neq b_{2} . \\
P_{e}^{1} P_{e}^{2} & \text { if } \hat{b}_{1} \neq b_{1}, \hat{b}_{2} \neq b_{2} .\end{cases}
\end{aligned}
$$

It should also be remarked that, since the proposed scheme is working on a single-slot basis, it is assumed that the decoding delay at the relay is negligible with respect to symbol time intervals. Therefore, the relay is able to start the retransmission of the detected symbol after some small delay during the same time slot.

\section{Analysis}

In the following section a detailed derivation of the analytic probability of error for both schemes is provided. We begin by analyzing the AF approach.

\subsection{Analysis of Amplify-and-Forward ML Detector at the Relay}

In order to compute the analytic probability of error for the AF approach, we first rewrite Equation (3) as the sum of a signal term $\mathbf{u}$ and a noise term $\mathbf{w}$, i.e. $\mathbf{y}=\mathbf{u}+\mathbf{w}$, where $\mathbf{u}$ is given by

$$
\mathbf{u}=\left(\begin{array}{l}
h_{1} b_{1}+\gamma_{1} h_{5} h_{6} b_{2} \\
\gamma_{2} h_{2} h_{3} b_{1}+h_{4} b_{2}
\end{array}\right)
$$

and the noise term $\mathbf{w}$ will be zero mean with a covariance expressed as ${ }^{1}$

$$
\mathrm{E}\left\{\mathbf{w} \mathbf{w}^{*}\right\}=\left(\begin{array}{cc}
N_{1} & 0 \\
0 & N_{2}
\end{array}\right)
$$

${ }^{1} \mathrm{E}\{\cdot\}$ denotes mathematical expectation of a random variable. where $N_{1} \square N_{0}\left(1+\gamma_{1}^{2}\left|h_{6}\right|^{2}\right)$ and $N_{2} \square N_{0}\left(1+\gamma_{2}^{2}\left|h_{3}\right|^{2}\right)$. Since the two terms of the noise vector $\mathbf{w}$ do not present the same variance, we will employ a scaling matrix $M$ so as to transform $\mathbf{w}$ into a unit variance vector $\mathbf{w}^{\prime}=M \mathbf{w}$ such that $\mathbf{E}\left\{\mathbf{w}^{\prime} \mathbf{w}^{\prime *}\right\}=\mathbf{I}_{2 \times 2}$, yielding

$$
M=\left(\begin{array}{cc}
\frac{1}{\sqrt{N_{1}}} & 0 \\
0 & \frac{1}{\sqrt{N_{2}}}
\end{array}\right) .
$$

As previously mentioned, the values of the relay gains $\gamma_{1}$ and $\gamma_{2}$ are set such that the average relay transmit power is normalized to one, yielding

$$
\gamma=\frac{1}{\sqrt{\left|h_{2}\right|^{2}+\left|h_{5}\right|^{2}}}
$$

We will henceforth denote the received vector corresponding to $\left(b_{1}, b_{2}\right)=(1,1) \square \mathbf{x}_{A}^{\mathrm{T}}$ by $\mathbf{u}_{A}$. Similarly, vectors $\mathbf{u}_{B}, \quad \mathbf{u}_{C}$, and $\mathbf{u}_{D}$ correspond to $\mathbf{x}_{B}^{\mathrm{T}} \square(-1,1)$, $\mathbf{x}_{C}^{\mathrm{T}} \square(1,-1)$ and $\mathbf{x}_{D}^{\mathrm{T}} \square(-1,-1)$, respectively. Assuming that the possible transmit vectors $\left(b_{1}, b_{2}\right)$ are equiprobable, the total probability of error is thus given by

$$
\begin{aligned}
P_{e} & =\frac{1}{4}\left(\operatorname { P r } \left\{\text { error } \mid\left(b_{1}, b_{2}\right)\right.\right. \\
& \left.=\mathbf{x}_{A}^{\mathrm{T}}\right\}+\operatorname{Pr}\left\{\operatorname{error} \mid\left(b_{1}, b_{2}\right)\right. \\
& \left.=\mathbf{x}_{B}^{\mathrm{T}}\right\}+\operatorname{Pr}\left\{\operatorname{error} \mid\left(b_{1}, b_{2}\right)\right. \\
& \left.\left.=\mathbf{x}_{C}^{\mathrm{T}}\right\}+\operatorname{Pr}\left\{\operatorname{error} \mid\left(b_{1}, b_{2}\right)=\mathbf{x}_{D}^{\mathrm{T}}\right\}\right) \\
& =\frac{1}{4} \sum_{i=A}^{D} \operatorname{Pr}\left\{\operatorname{error} \mid\left(b_{1}, b_{2}\right)\right. \\
& \left.=\mathbf{x}_{i}^{\mathrm{T}}\right\} .
\end{aligned}
$$

Let us consider the error term corresponding to $\mathbf{x}_{A}^{\mathrm{T}}$. Given a set of channel coefficients $\left\{h_{i}\right\}_{i=1}^{6}$, we will use the union bound to compute the probability of error when $\mathbf{x}_{A}^{\mathrm{T}}$ was sent, resulting in

$$
\begin{aligned}
\operatorname{Pr} & \left\{\operatorname{error} \mid\left(b_{1}, b_{2}\right)=\mathbf{x}_{A}^{\mathrm{T}},\left\{h_{i}\right\}_{i=1}^{6}\right\} \\
& \leq \operatorname{Pr}\left\{\mathbf{x}_{A} \rightarrow \mathbf{x}_{B} \mid\left\{h_{i}\right\}_{i=1}^{6}\right\} \\
& +\operatorname{Pr}\left\{\mathbf{x}_{A} \rightarrow \mathbf{x}_{C} \mid\left\{h_{i}\right\}_{i=1}^{6}\right\} \\
& +\operatorname{Pr}\left\{\mathbf{x}_{A} \rightarrow \mathbf{x}_{D} \mid\left\{h_{i}\right\}_{i=1}^{6}\right\}
\end{aligned}
$$

where $\operatorname{Pr}\left\{\mathbf{x}_{i} \rightarrow \mathbf{x}_{j} \mid\left\{h_{i}\right\}_{i=1}^{6}\right\}$ denotes the probability that, given a set of channel coefficients $\left\{h_{i}\right\}_{i=1}^{6}, \mathbf{x}_{i}$ is transmitted and $\mathbf{x}_{j}$ is detected at the receiver. Let us consider the term $\operatorname{Pr}\left\{\mathbf{x}_{A} \rightarrow \mathbf{x}_{B} \mid\left\{h_{i}\right\}_{i=1}^{6}\right\}$. This pairwise probability of 
error can be obtained by using the transformation in Expression (12), yielding

$$
\begin{aligned}
\operatorname{Pr} & \left\{\mathbf{x}_{i} \rightarrow \mathbf{x}_{j} \mid\left\{h_{i}\right\}_{i=1}^{6}\right\} \\
& =Q\left(\frac{\left\|M\left(\mathbf{u}_{A}-\mathbf{u}_{B}\right)\right\|}{\sqrt{2}}\right) \\
& =Q\left(\sqrt{2\left(\frac{\left|h_{1}\right|^{2}}{N_{1}}+\frac{\gamma^{2}\left|h_{2}\right|^{2}\left|h_{3}\right|^{2}}{N_{2}}\right)}\right)
\end{aligned}
$$

where $\|\cdot\|$ denotes the Frobenius norm. Consequently, the union bound for the probability of error $\operatorname{Pr}\left\{\operatorname{error} \mid\left(b_{1}, b_{2}\right)=\mathbf{x}_{A}^{\mathrm{T}}\right\} \quad$ can be computed by adding the other terms in Expression (15) and taking the expectation over channel coefficients $h_{i}$, i.e.

$$
\begin{aligned}
& \operatorname{Pr}\left\{\operatorname{error} \mid\left(b_{1}, b_{2}\right)=\mathbf{x}_{A}^{\mathrm{T}}\right\} \\
& \leq \mathrm{E}_{h_{i}}\left\{Q\left(\sqrt{2\left(\frac{\left|h_{1}\right|^{2}}{N_{1}}+\frac{\gamma^{2}\left|h_{2}\right|^{2}\left|h_{3}\right|^{2}}{N_{2}}\right)}\right)\right. \\
& +Q\left(\sqrt{2\left(\frac{\gamma^{2}\left|h_{5}\right|^{2}\left|h_{6}\right|^{2}}{N_{1}}+\frac{\left|h_{4}\right|^{2}}{N_{2}}\right)}\right) \\
& \left.+Q\left(\sqrt{2\left(\frac{\left|h_{1}+\gamma h_{5} h_{6}\right|^{2}}{N_{1}}+\frac{\left|h_{4}+\gamma h_{2} h_{3}\right|^{2}}{N_{2}}\right)}\right)\right\}
\end{aligned}
$$

Finally, it can be easily verified that the other terms in Expression (14) can be upper-bounded by a similar expression to that corresponding to $\operatorname{Pr}\left\{\operatorname{error} \mid\left(b_{1}, b_{2}\right)=\mathbf{x}_{A}^{\mathrm{T}}\right\}$. Therefore, the overall probability of error will be given by $P_{e}=\operatorname{Pr}\left\{\right.$ error $\left.\mid\left(b_{1}, b_{2}\right)=\mathbf{x}_{A}^{\mathrm{T}}\right\}$, i.e. the same bound given in Equation (17) will also be valid for the end-to-end probability of error $P_{e}$. As verified by our simulation results, this expression results in a tight upper-bound of the end-to-end probability of error for the AF approach.

\subsection{Analysis of Detect-and-Forward ML Detector at the Relay}

Equations (4) and (5) show that the analysis of the DF method is quite different than that of the AF approach. In the DF case we have complex Gaussian noise of the same variance for both components of the received vector $\mathbf{y}$ and, since $\hat{b}_{1}, \hat{b}_{2} \in\{-1,1\}$, the relay gain factor is set to $\frac{1}{\sqrt{2}}$, which is not a function of channel variables. However, the computation of the analytic probability of error for the former is more complicated since the detected bit values of $\hat{b}_{1}$ and $\hat{b}_{2}$ at the relay are random variables that depend on the condition of the channel from the sources to the relay. In fact, when the vector $\mathbf{x}_{A}^{\mathrm{T}}=(1,1)$ is transmitted from the sources, the noise-free signal $\mathbf{u}_{\mathrm{A}}$ received at the destination may be any of the following set with the corresponding probability,

$$
\mathbf{u}_{A}=\left\{\begin{array}{l}
\left(\begin{array}{l}
h_{1}+\gamma h_{6} \\
h_{4}+\gamma h_{3}
\end{array}\right) \square \mathbf{u}_{A 1}, \operatorname{Pr}\left\{\mathbf{u}=\mathbf{u}_{A 1}\right\}=\left(1-P_{e}^{1}\right)\left(1-P_{e}^{2}\right) \\
\left(\begin{array}{l}
h_{1}+\gamma h_{6} \\
h_{4}-\gamma h_{3}
\end{array}\right) \square \mathbf{u}_{A 2}, \operatorname{Pr}\left\{\mathbf{u}=\mathbf{u}_{A 2}\right\}=P_{e}^{1}\left(1-P_{e}^{2}\right) \\
\left(\begin{array}{l}
h_{1}-\gamma h_{6} \\
h_{4}+\gamma h_{3}
\end{array}\right) \square \mathbf{u}_{A 3}, \operatorname{Pr}\left\{\mathbf{u}=\mathbf{u}_{A 3}\right\}=\left(1-P_{e}^{1}\right) P_{e}^{2} \\
\left(\begin{array}{l}
h_{1}-\gamma h_{6} \\
h_{4}-\gamma h_{3}
\end{array}\right) \square \mathbf{u}_{A 4}, \operatorname{Pr}\left\{\mathbf{u}=\mathbf{u}_{A 4}\right\}=P_{e}^{1} P_{e}^{2}
\end{array}\right.
$$

where $P_{e}^{1}$ and $P_{e}^{2}$ are given in Expressions (6) and (7), respectively. Similarly, if the transmit vector $\mathbf{x}_{B}^{\mathrm{T}}=(-1,1)$ is sent, the possible received signal set will be

$$
\mathbf{u}_{A}=\left\{\begin{array}{l}
\left(\begin{array}{c}
-h_{1}+\gamma h_{6} \\
h_{4}-\gamma h_{3}
\end{array}\right) \square \mathbf{u}_{B 1}, \operatorname{Pr}\left\{\mathbf{u}=\mathbf{u}_{B 1}\right\}=\left(1-P_{e}^{1}\right)\left(1-P_{e}^{2}\right) \\
\left(\begin{array}{c}
-h_{1}+\gamma h_{6} \\
h_{4}+\gamma h_{3}
\end{array}\right) \square \mathbf{u}_{B 2}, \operatorname{Pr}\left\{\mathbf{u}=\mathbf{u}_{B 2}\right\}=P_{e}^{1}\left(1-P_{e}^{2}\right) \\
\left(\begin{array}{c}
-h_{1}-\gamma h_{6} \\
h_{4}-\gamma h_{3}
\end{array}\right) \square \mathbf{u}_{B 3}, \operatorname{Pr}\left\{\mathbf{u}=\mathbf{u}_{B 3}\right\}=\left(1-P_{e}^{1}\right) P_{e}^{2} \\
\left(\begin{array}{c}
-h_{1}-\gamma h_{6} \\
h_{4}+\gamma h_{3}
\end{array}\right) \square \mathbf{u}_{B 4}, \operatorname{Pr}\left\{\mathbf{u}=\mathbf{u}_{B 4}\right\}=P_{e}^{1} P_{e}^{2}
\end{array}\right.
$$

From the above definitions, the probability that $\mathbf{x}_{A}$ is transmitted and $\mathbf{x}_{B}$ is detected will be then given by the probability that one of the four signals corresponding to $\mathbf{x}_{A}$ is transmitted and one of the signals corresponding to $\mathbf{x}_{B}$ is detected. Since we have four different transmit pairs $\left(b_{1}, b_{2}\right)$ (each of which can be transmitted in four different ways from the relay), an exact computation of the probability of error at the destination will involve complex scenarios of non-uniform vector constellations with nonuniform probabilities. To simplify this computation, we will derive an approximate probability of error by solely accounting for the most probable error events in our scenario.

Having said this, we will consider two main terms in the probability of error for the case when $\mathbf{x}_{A}$ is sent, i.e. $\operatorname{Pr}\left\{\right.$ error $\left.\mid\left(b_{1}, b_{2}\right)=\mathbf{x}_{A}^{\mathrm{T}}\right\}$. The first component, denoted by $P_{e,}$, corresponds to the case that no error has occurred at the relay, and therefore the signal $\mathbf{u}_{A 1}$ with probability $\operatorname{Pr}\left\{\mathbf{u}_{A}=\mathbf{u}_{A 1}\right\}$ is transmitted among all the points associated to $\mathbf{u}_{A}$. We will then compute the probability that this signal is detected erroneously as one of the three most likely points associated to $\mathbf{u}_{B}$ (i.e. ignoring the point $\mathbf{u}_{B 4}$ with much smaller probability $\left.\operatorname{Pr}\left\{\mathbf{u}_{B}=\mathbf{u}_{B 4}\right\}\right)$. 
Consequently, $P_{e, \diamond}$ can be approximated as

$$
\begin{aligned}
P_{e, \diamond} & \square \operatorname{E}\left\{\operatorname{Pr}\left\{\mathbf{u}=\mathbf{u}_{A 1}\right\}\right\} \mathrm{E}\left\{Q\left(\frac{\sqrt{\left|h_{1}\right|^{2}+\left|h_{3}\right|^{2}}}{\sqrt{2} \sigma}\right)\right. \\
& +Q\left(\frac{\sqrt{\left|h_{1}\right|^{2}}}{\sigma}+\frac{\sigma \ln \frac{\operatorname{Pr}\left\{\mathbf{u}=\mathbf{u}_{A 1}\right\}}{\operatorname{Pr}\left\{\mathbf{u}=\mathbf{u}_{B 2}\right\}}}{2 \sqrt{\left|h_{1}\right|^{2}}}\right) \\
+ & Q\left(\frac{\left.\sqrt{\left|h_{1}\right|^{2}+\frac{\left|h_{3}\right|^{2}+\left|h_{6}\right|^{2}}{2}+\sqrt{2} \Re\left(h_{1} h_{6}^{*}\right.}\right)}{\sigma}\right) \\
+ & \left.\left.\frac{\sigma \ln \frac{\operatorname{Pr}\left\{\mathbf{u}=\mathbf{u}_{A 1}\right\}}{\operatorname{Pr}\left\{\mathbf{u}=\mathbf{u}_{B 3}\right\}}}{2 \sqrt{\left|h_{1}\right|^{2}+\frac{\left|h_{3}\right|^{2}+\left|h_{6}\right|^{2}}{2}+\sqrt{2} \Re\left(h_{1} h_{6}^{*}\right)}}\right)\right\}
\end{aligned}
$$

where $\mathfrak{R}(\cdot)$ stands for the real part of a complex value, and $\sigma^{2}$ denotes the variance per dimension of the noise term $\mathrm{n}=\left(n_{3}, n_{4}\right)^{\mathrm{T}}$ at the destination. Observe that the additional factors $\ln \frac{\operatorname{Pr}\left\{\mathbf{u}=\mathbf{u}_{A 1}\right\}}{\operatorname{Pr}\left\{\mathbf{u}=\mathbf{u}_{B 2}\right\}}$ and $\ln \frac{\operatorname{Pr}\left\{\mathbf{u}=\mathbf{u}_{A 1}\right\}}{\operatorname{Pr}\left\{\mathbf{u}=\mathbf{u}_{B 3}\right\}}$ in the second and third terms of the above equation are due to the non-equal probabilities of occurrence of the constellation vectors $\mathbf{u}_{A 1}, \mathbf{u}_{B 2}$ and $\mathbf{u}_{B 3}$.

The second main component of the proposed approximation for $\operatorname{Pr}\left\{\operatorname{error} \mid\left(b_{1}, b_{2}\right)=\mathbf{x}_{A}^{\mathrm{T}}\right\}$, denoted as $P_{e, \square}$, is related to the case when the relay has wrongly detected the bit values $(-1,1)$ corresponding to the point $\mathbf{u}_{A 2}$. Therefore, we must compute the probability that this signal, which belongs to the set associated to $\mathbf{X}_{A}$, is erroneously detected as $\mathbf{X}_{B}$ at destination. In this case, such an error probability can be approximated by the probability that this signal is transmitted by the relay and is detected as the point $\mathbf{u}_{B 1}$, which presents the highest probability among all the points associated to $\mathbf{u}_{B}$. In other words, an error event will occur if the received signal $\mathrm{y}=\mathbf{u}_{A 2}+\mathbf{n}$ is more likely to be detected as $\mathbf{u}_{B 1}$ instead of $\mathbf{u}_{A 2}$ or $\mathbf{u}_{A 1}$. At this point it should be noted that, since we assume that the signal $\mathbf{u}_{A 2}$ is transmitted from the relay, the probability of detecting the less probable constellation points $\mathbf{u}_{A 3}$ and $\mathbf{u}_{A 4}$ is negligible in comparison with the above mentioned probabilities. Thus the second error term $P_{e, \square}$ can be approximated by

$$
\begin{aligned}
& P_{e, \square} \square \mathrm{E}\left\{\operatorname { P r } \left(-\left\|\left(\mathbf{u}_{A 2}+\mathbf{n}\right)-\mathbf{u}_{B 1}\right\|^{2}>-\left\|\left(\mathbf{u}_{A 2}+\mathbf{n}\right)-\mathbf{u}_{A 1}\right\|^{2},\right.\right. \\
& \left.\left.-\left\|\left(\mathbf{u}_{A 2}+\mathbf{n}\right)-\mathbf{u}_{B 1}\right\|^{2}>-\left\|\left(\mathbf{u}_{A 2}+\mathbf{n}\right)-\mathbf{u}_{A 2}\right\|^{2}\right)\right\}
\end{aligned}
$$

The above joint probability can be computed by considering the projections of the noise term $\mathbf{n}$ on the two directions $\mathbf{u}_{B 1}-\mathbf{u}_{A 1}$ and $\mathbf{u}_{B 1}-\mathbf{u}_{A 2}$, and integrating over the joint probability distribution of these two projection terms. Let us denote the correlation factor of these two noise terms as

$$
\begin{aligned}
\rho & \square \frac{\left\langle\mathbf{u}_{B 1}-\mathbf{u}_{A 2}, \mathbf{u}_{B 1}-\mathbf{u}_{A 1}\right\rangle}{\left\|\mathbf{u}_{B 1}-\mathbf{u}_{A 2}\right\| \cdot\left\|\mathbf{u}_{B 1}-\mathbf{u}_{A 1}\right\|} \\
& =\frac{4\left|h_{1}\right|^{2}}{2\left|h_{1}\right| \cdot 2 \sqrt{\left|h_{1}\right|^{2}+\gamma^{2}\left|h_{3}\right|^{2}}},
\end{aligned}
$$

where $\langle\cdot$,$\rangle denotes the inner product of two vectors.$ With this definition, Expression (21) reduces to [27]

$$
P_{e, \square} \square \mathrm{E}\left\{\frac{1}{2 \pi \sqrt{1-\rho^{2}}} \int_{a}^{\infty} \int_{b}^{\infty} e^{\frac{-\left(x^{2}-2 \rho x y+y^{2}\right)}{2\left(1-\rho^{2}\right)}} d x d y\right\}
$$

where the integration limits $a$ and $b$ are obtained by computing the distance of the received signal from the decision boundaries between $\mathbf{u}_{B 1}$ and $\mathbf{u}_{A 1}$ and between $\mathbf{u}_{B 2}$ and $\mathbf{u}_{A 2}$, respectively. Further geometric manipulations lead to

$$
\begin{aligned}
a & =\frac{\left\|\mathbf{u}_{B 1}\right\|^{2}-\left\|\mathbf{u}_{A 1}\right\|^{2}+2<\mathbf{u}_{A 2}, \mathbf{u}_{A 1}-\mathbf{u}_{B 1}>}{2 \sigma\left\|\mathbf{u}_{B 1}-\mathbf{u}_{A 1}\right\|} \\
& =\frac{\sqrt{\left|h_{1}\right|^{2}+\gamma^{2}\left|h_{3}\right|^{2}}}{\sigma}-\frac{4 \gamma^{2}\left|h_{3}\right|^{2}}{2 \sigma \sqrt{\left|h_{1}\right|^{2}+\gamma^{2}\left|h_{3}\right|^{2}}} \\
b & =\frac{\left\|\mathbf{u}_{A 2}-\mathbf{u}_{B 1}\right\|}{2 \sigma}+\frac{\sigma}{\left\|\mathbf{u}_{A 2}-\mathbf{u}_{B 1}\right\|} \ln \frac{\operatorname{Pr}\left\{\mathbf{u}=\mathbf{u}_{A 2}\right\}}{\operatorname{Pr}\left\{\mathbf{u}=\mathbf{u}_{B 1}\right\}} \\
& =\frac{\sqrt{\left|h_{1}\right|^{2}}}{\sigma}+\frac{\sigma}{2 \sqrt{\left|h_{1}\right|^{2}}} \ln \frac{\operatorname{Pr}\left\{\mathbf{u}=\mathbf{u}_{A 2}\right\}}{\operatorname{Pr}\left\{\mathbf{u}=\mathbf{u}_{B 1}\right\}} .
\end{aligned}
$$

It should be noted that, when computing $P_{e, \diamond}$, the value of $\operatorname{Pr}\left\{\mathbf{u}=\mathbf{u}_{B 2}\right\}=P_{e}^{1}\left(1-P_{e}^{2}\right)$ should be obtained based on channel values that cause errors at the relay. Analogously, $P_{e, \square}$ should be computed by averaging over all values of $h_{2}$ that cause such errors at the relay, and not over the whole range of $h_{2}$. Finally, the approximate end-to-end probability of error $P_{\mathrm{e}}$ for the DF scheme can be obtained by adding these two main error terms, i.e.

$$
P_{e} \square P_{e, \diamond}+P_{e, \square}
$$

which, as shown in next section, is in close match with the Monte Carlo simulation results.

\section{Simulation Results}

In this section we provide Monte Carlo simulation results for the proposed algorithm in comparison with the previously derived analytic approximations. The simulations 
have been performed assuming BPSK signaling (i.e. $b_{1}, b_{2}$ $\in\{-1,1\})$. The transmitted signals at the sources and the relay are assumed to be of unit average power. Flat Rayleigh fading coefficients were generated based on a complex Gaussian distribution with variance per 2 dimensions equal to one. As mentioned earlier, an additional signal power attenuation equal to $K / d^{2}$ was assumed for signal propagation over a distance equal to $d$, where $K$ is the propagation factor (set to $10^{-4}$ in our simulations). Fading coefficients have been assumed to be constant over a block of 100 samples, and are independently generated over different blocks. The Signal to Noise Ratio (SNR) is defined as the ratio of the average received power of the direct source-destination path of one of the sources (where average transmit power is assumed to be equal to one) to the noise variance per dimension. The noise variance used for computing the SNR has been assumed to be the same at the relay and destination sites. Furthermore, the relay gains $\gamma_{1}$ and $\gamma_{2}$ have been chosen such that average transmit power of the relay is also normalized to one. Finally, in order to investigate the effect of the relay location on the performance of the different considered schemes, the location of the relay varies over a range of $d=0$ to $d=1$. The results for $d=0.1$ (relay close to sources) and $d=0.9$ (relay close to destination) are shown in these plots.

Figure 2 depicts the probability of symbol error of the proposed $\mathrm{DF}$ and $\mathrm{AF}$ algorithms versus the SNR ratio at two different relay locations. First notice that in both approaches the obtained analytic approximation for the probability of error is in close match with the corresponding simulated curves. Also observe that the performance of the DF approach even improves slightly as the relay position is changed from a location close to sources $(d=0.1)$ to a location close to the destination $(d=0.9)$. However, the performance degrades considerably for the AF scheme as the distance between the relay and the sources increases. Based on these results it is foreseen that, in scenarios where the relay is close to the destination, the use of the proposed switched-frequency

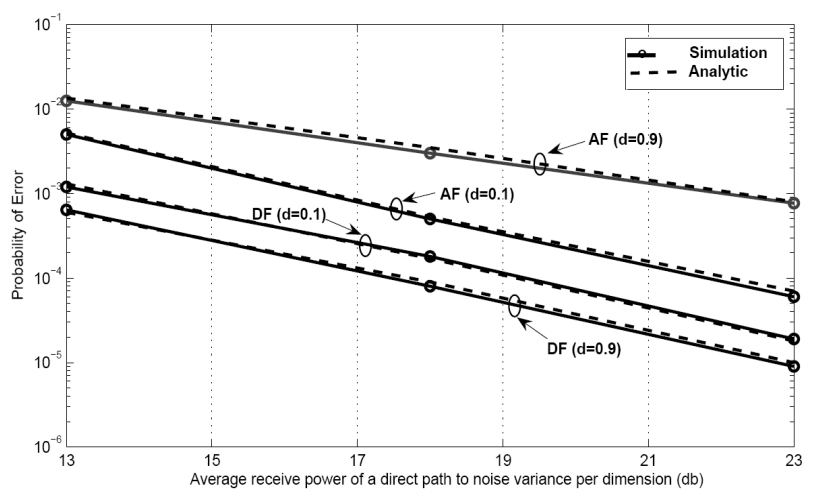

Figure 2. Analytic and simulated probability of symbol error for the proposed frequency switching AF and DF schemes at two different relay positions.
DF scheme will yield a significant performance enhancement (around $10 \mathrm{~dB}$ in our proposed setup) at the cost of a minor complexity increase.

\section{Concluding Remarks}

In this paper, the error performance of a novel communication scheme for the two-user single-relay multiple access channel has been proposed, which achieves a diversity of order two by using only two frequency channels over all the links. The main advantage of the proposed scheme is to obviate the requirement of complex superposition-type coding schemes in Detect-and-Forward scenarios. The effect of the relay location for both $\mathrm{AF}$ and DF schemes has also been investigated, concluding in a superior performance of the DF scheme as the relay gets closer to the destination.

\section{Acknowledgements}

This work was supported in part by the Spanish Ministry of Science and Innovation through the CONSOLIDER-INGENIO 2010 programme (CSD2008-00010, www.comonsens.org), and by the Basque Government through the Future Internet project (ETORTEK programme).

\section{References}

[1] C. T. K. Ng and A. J. Goldsmith, "Capacity and cooperation in wireless networks," in Proceedings of Information Theory and Applications (ITA) Workshop, February 2006.

[2] _ - "The impact of CSI and power allocation on relay channel capacity and cooperation strategies," submitted to IEEE Transactions on Information Theory, January 2007.

[3] S. Avestimehr and D. Tse, "Outage capacity of the fading relay channel in the low SNR regime," IEEE Transactions on Information Theory, Vol. 53, No. 4, pp 1401-1415, April 2007.

[4] R. U. Nabar and H. Kneubuhler, F.W.; Bolcskei, "Performance limits of amplify-and-forward based fading relay channels," in Proceedings of IEEE International Conference on Audio, Speech and Signal Processing (ICASSP), May 2004.

[5] C. Hausl and P. Dupraz, "Joint network-channel coding for the multiple-access relay channel," in Proceedings of International Workshop on Wireless Ad Hoc and Sensor Networks (IWWAN), June 2006.

[6] J. Del Ser, P. M. Crespo, B. H. Khalaj, and J. Gutierrez-Gutierrez, "On combining distributed joint source-channel-network coding and turbo equalization in multiple access relay networks," in Proceedings of 3rd IEEE International Conference on Wireless and Mobile 
Computing, Networking and Communications (WiMob' 07), October 2007.

[7] G. Kramer and A. J. van Wijngaarden, "On the white gaussian multiple access relay channel," in Proceedings of the International Symposium on Information Theory, June 2000.

[8] L. Sankaranarayanan, G. Kramer, and N. B. Mandayam, "Capacity theorems for the multiple-access relay channel," in Proceedings of the 42nd Annual Allerton Conference on Communication, Control, and Computing, October 2004.

[9] A. Sendonaris, E. Erkip, and B. Aazhang, "User cooperation diversity - Part I: System description," IEEE Transactions on Communications, Vol. 51, No. 11, pp. 1927-1938, November 2003.

[10] T. E. Hunter and A. Nosratinia, "Cooperation diversity through coding," in Proceedings of the IEEE International Symposium on Information Theory, 2002.

[11] J. N. Laneman, D. N. C. Tse, and G. W. Wornell, "Cooperative diversity in wireless networks: Efficient protocols and outage behavior," IEEE Transactions on Information Theory, Vol. 50, No. 12, pp. 3062-3080, December 2004.

[12] S. Valentin, H. S. Lichte, H. Karl, G. Vivier, S. Simoens, J. Vidal, A. Agustin, and I. Aad, "Cooperative wireless networking beyond store-and-forward: Perspectives for PHY and MAC design," in Proceedings of the 17th Wireless World Research Forum Meeting (WWRF 17), November 2006.

[13] P. Herhold, E. Zimmermann, and G. Fettweis, "On the performance of cooperative amplify-and-forward relay networks," in Proceedings of the 5th International ITG Conference on Source and Channel Coding (SCC), January 2004.

[14] K. Azarian, H. E. Gamal, and P. Schniter, "On the achievable diversity-multiplexing tradeoff in half-duplex cooperative channels," IEEE Transactions on Information Theory, Vol. 51, No. 12, pp. 4152-4172, December 2005.

[15] M. Yu and J. T. Li, "Is amplify-and-forward practically better than decode-and-forward or viceversa?" in Proceedings of the IEEE International Conference on Audio, Speech and Signal Processing (ICASSP), Vol. 3, pp. 365-368, March 2005.

[16] N. Seshadri and J. H. Winters, “Two signaling schemes for improving the error performance of frequency-division-multiplex (FDD) transmission systems using transmitter antenna diversity," in Proceedings of the IEEE Vehicular Technology Conference, Vol. 1, pp. 508-511,
May 1993.

[17] A. Wittneben, "A new bandwidth efficient transmit antenna modulation diversity scheme for linear digital modulation," in Proceedings of IEEE International Conference on Communications, Vol. 3, pp. 1630-1634, May 1993.

[18] V. Tarokh, N. Seshadri, and A. Calderbank, "Space-time codes for high data rate wireless communication: Performance analysis and code construction," IEEE Transactions on Information Theory, Vol. 44, No. 3, pp. 744-765, March 1998.

[19] D. Chen, K. Azarian, and J. N. Laneman, "A case for amplify-forward relaying in the block-fading multiaccess channel," submitted to IEEE Transactions on Information Theory, January 2007.

[20] L. Sankar, G. Kramer, and N. B. Mandayam, "Offset encoding for multiple access relay channels," IEEE Transactions on Information Theory, Vol. 53, No. 10, pp. 3814-3821, October 2007.

[21] L. Sankar, Y. Linang, H. V. Poor, and N. Mandayam, "Opportunistic communication in an orthogonal multiaccess relay channel," in Proceedings of the International Symposium on Information Theory, June 2007.

[22] B. H. Khalaj, J. Del Ser, P. M. Crespo, and J. GutierrezGutierrez, "A novel dual-frequency multiple access relay transmission scheme," in Proceedings of IEEE International Symposium on Personal, Indoor, and Mobile Radio Communications (IEEE PIMRC), September 2007.

[23] B. Sklar, "Rayleigh fading channels in mobile digital communication systems Part I: Characterization," IEEE Communications Magazine, Vol. 35, No. 7, pp. 90-100, July 1997.

[24] H. Ju, S. Lee, K. Kwak, E. Oh, and D. Hong, "A new duplex without loss of data rate and utilizing selection diversity," in Proceedings of IEEE Vehicular Technology Conference, pp. 1519-1523, May 2008.

[25] C. Hausl, F. Schreckenbach, I. Oikonomidis, and G. Bauch, "Iterative network and channel decoding on a tanner graph," in Proceedings of 43rd Allerton Conference on Communication, Control, and Computing, September 2005.

[26] H. Jafarkhani, "Space-time coding, theory and practice," Cambridge University Press, 2005.

[27] H. Kuai, F. Alajaji, and G. Takahara, "Tight error bounds for nonuniform signaling over AWGN channels," IEEE Transactions on Information Theory, Vol. 46, No. 7, pp. 2712-2718, November 2000. 